\title{
Human Spaceflight Technology Needs-a Foundation for JSC's Technology Strategy
}

\author{
Jonette M. Stecklein \\ NASA Johnson Space Center \\ 2101 NASA Parkway, MC YX \\ Houston, TX 77058 \\ 281-244-7146 \\ Jonette.M.Stecklein@nasa.gov
}

\begin{abstract}
Human space exploration has always been heavily influenced by goals to achieve a specific mission on a specific schedule. This approach drove rapid technology development, the rapidity of which adds risks as well as provides a major driver for costs and cost uncertainty. The National Aeronautics and Space Administration (NASA) is now approaching the extension of human presence throughout the solar system by balancing a proactive yet less schedule-driven development of technology with opportunistic scheduling of missions as the needed technologies are realized. This approach should provide cost effective, low risk technology development that will enable efficient and effective manned spaceflight missions.
\end{abstract}

As a first step, the NASA Human Spaceflight Architecture Team (HAT) has identified a suite of critical technologies needed to support future manned missions across a range of destinations, including in cis-lunar space, near earth asteroid visits, lunar exploration, Mars moons, and Mars exploration. The challenge now is to develop a strategy and plan for technology development that efficiently enables these missions over a reasonable time period, without increasing technology development costs unnecessarily due to schedule pressure, and subsequently mitigating development and mission risks.

NASA's Johnson Space Center (JSC), as the nation's primary center for human exploration, is addressing this challenge through an innovative approach in allocating Internal Research and Development funding to projects. The HAT Technology Needs (TechNeeds) Database has been developed to correlate across critical technologies and the NASA Office of Chief Technologist Technology Area Breakdown Structure (TABS). The TechNeeds Database illuminates that many critical technologies may support a single technical capability gap, that many HAT technology needs may map to a single TABS technology discipline, and that a single HAT technology need may map to multiple TABS technology disciplines. The TechNeeds Database greatly clarifies understanding of the complex relationships of critical technologies to mission and architecture element needs. Extensions to the core TechNeeds Database allow JSC to factor in and appropriately weight JSC Center Core Technology Competencies, and considerations of Commercialization Potential and Partnership Potential. The inherent coupling among these, along with an appropriate importance weighting, has provided an initial prioritization for allocation of technology development research funding for JSC.

The HAT Technology Needs Database, with a core of built-in reports, clarifies and communicates complex technology needs for cost effective human space exploration such that an organization seeking to assure that research prioritization supports human spaceflight of the future can be successful.

U.S. Government work not protected by U.S. copyright

\section{TABLE OF CONTENTS}

1. INTRODUCTION ............................................1

2. BACKGROUND ...............................................2

Elements and Destinations............................... 2

Technology Needs .......................................... 4

3. Challenges For JSC TEChNOLOGY

PRIORITIZATION............................................4

4. HAT TECHNOLOGY NEEDS DATABASE ..........4

5. JSC TeChNology NeEds Prioritization ..6

6. SUMMARY ...................................................8

ACKNOWLEDGEMENTS......................................9

REFERENCES..........................................................99

BIOGRAPHY ...................................................99

APPENDIX A: HAT TECHNOLOGY NEEDS

MATCHED TO JSC TECHNOLOGY

COMPETENCIES ............................................10

APPENDIX B: ACRONYMS .................................24

\section{INTRODUCTION}

NASA is preparing for the next chapter of space exploration by developing the capabilities needed to expand human activity throughout the inner solar system. [1] NASA formed the Human Spaceflight Architecture Team (HAT) to develop concepts for architectures and vehicle elements, conduct trade studies, and determine the technology and capability requirements needed for missions ranging from activities in cis-Lunar space to Mars landings. These activities provide cost and feasibility determinations to plan the next series of human exploration missions.

Determining a strategy for allocating funding for the technology developments most cost effectively, and to assure that initial mission opportunities are not missed, is a daunting challenge.

This paper describes an innovative process to accomplish this using a database that relates the complex issues associated with developing such a strategy. Section 2 is a synopsis of the efforts by the HAT to assess the possible design reference missions, determine the capability gaps, and identify the technology advancement needs that enable the future manned space exploration missions. Section 3 describes the challenges faced by JSC in developing a 
strategy to allocate funding for Internal Research and Development (IR\&D) and Innovative Charge Account (ICA) projects. Section 4 describes the HAT Technology Needs (TechNeeds) Database that was developed to enable a cost and mission effective funding allocation strategy. Section 5 explains how the TechNeeds Database was used by JSC with extensions that reflected JSC Center strategies and values. Section 6 summarizes the paper concluding with examples of how other organizations are using the TechNeeds Database, extending it for their particular needs.

\section{BACKGROUND}

As shown in Figure 1, the HAT approach includes several processes: design reference missions consistent with NASA's investment strategy are proposed; elements needed for the missions are conceptualized; schedule and cost estimates for each element are developed; integrated schedules and flight manifests are determined; and total costs are estimated. A key step in this process is the determination of which technologies are needed to enable these elements and missions so that full costs can be estimated.

The HAT created a Technology Development Assessment Team to manage the collection and evaluation of these technology needs. This team is comprised of representatives from across the Agency, ensuring input from and communication to a broad portion of the NASA community.

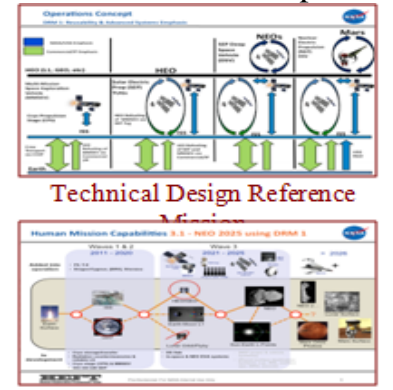

Investment strategy

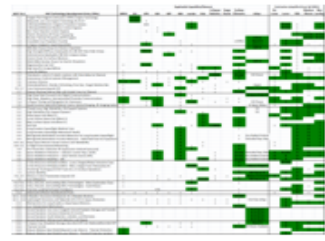

Technology Assessment
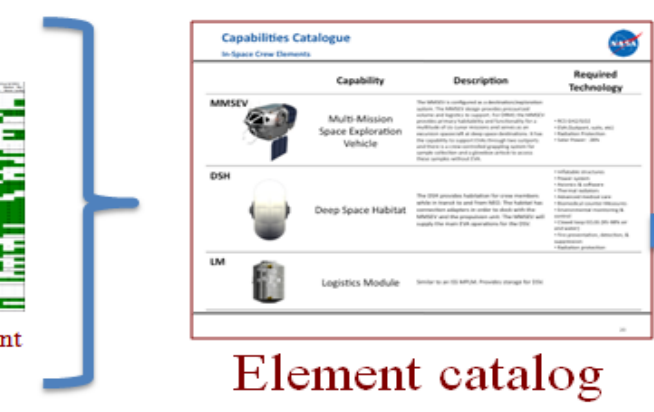

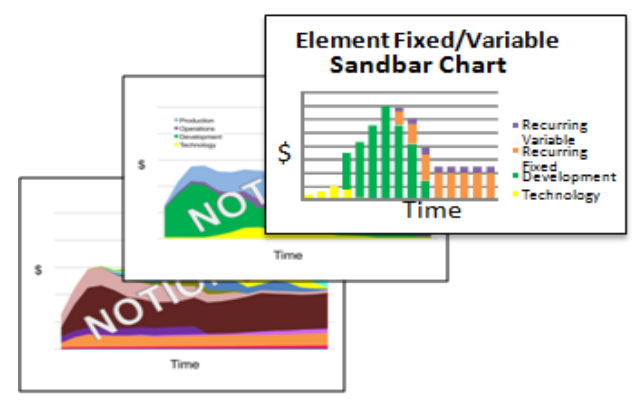

Cost Products

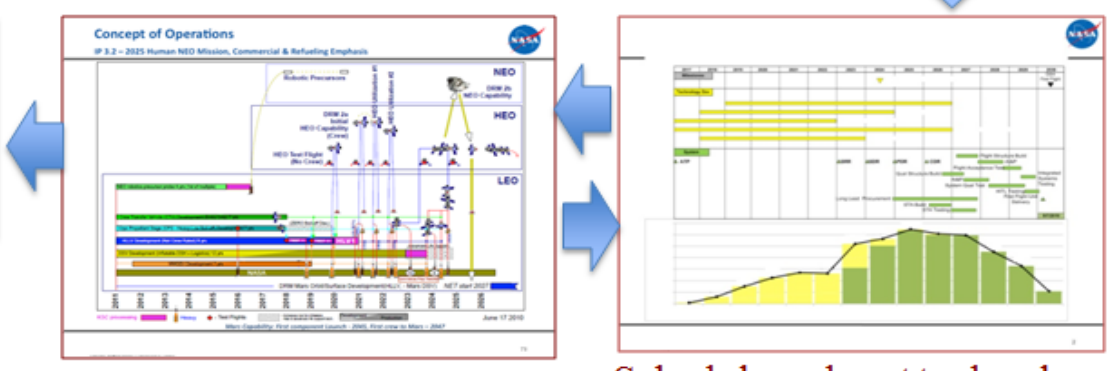

Integrated program schedule \& flight manifest
Schedule and cost to develop and operate each element

Figure 1: HAT Analysis Approach [2]

\section{Elements and Destinations}

Several architectural elements have been conceptualized by the HAT team, and many design reference missions have been developed to encompass a variety of destinations within the inner solar system. While still notional, these elements and missions contain enough fidelity to provide a concrete target for assessing the likely costs of similar missions, and to estimate the needs for technology development. The destinations are used to drive transportation systems capabilities and assess impacts of changes in mission assumptions. The elements and destinations currently under consideration are listed below in Table 1 and notional representations of the elements are illustrated in Figure 2. 
Table 1. HAT Identified Human Spaceflight Mission Architecture Elements and Missions/Destinations

\begin{tabular}{|c|c|}
\hline Architecture Elements [3] & $\begin{array}{c}\text { Design Reference Missions (DRM)/Destinations } \\
{[2][4] \text { [5] [6] [7] [8] }}\end{array}$ \\
\hline Space Launch System (SLS) & Low Earth Orbit (LEO) \\
\hline Multipurpose Crew Vehicle (MPCV) & $\begin{array}{l}\text { Geosynchronous and High Earth Orbit (GEO and } \\
\text { HEO) }\end{array}$ \\
\hline Cryogenic Propulsion Stage (CPS) & $\begin{array}{l}\text { Lunar Vicinity: Earth-Moon Lagrange points one } \\
\text { and two (E-M L1 and L2) }\end{array}$ \\
\hline Solar Electric Propulsion Stage (SEP) & Lunar flyby and Low Lunar Orbit (LLO) \\
\hline Lander & Lunar surface \\
\hline EVA Suit (EVA) & $\begin{array}{l}\text { Minimum capability, low energy Near Earth } \\
\text { Asteroid (NEA) }\end{array}$ \\
\hline Space Exploration Vehicle (SEV) & Full capability, high energy NEA \\
\hline Deep Space Habitat (DSH) & Mars moons \\
\hline In-Space Robotics & \multirow[t]{3}{*}{ Mars surface } \\
\hline Cargo Hauler & \\
\hline $\begin{array}{l}\text { Surface Elements (lunar, asteroid, Mars, and Mars } \\
\text { moons) }\end{array}$ & \\
\hline
\end{tabular}

The multipurpose crew vehicle and space launch system are needed for every destination, but the need for the other elements are destination and mission specific. Similarly, these two elements can be built with existing technology, but technology development is required for the other element concepts. For a mapping of technology needs to the elements and destinations that are enabled by the technology maturation, see [9].

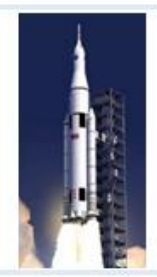

Space Launch System (SLS)HLLV

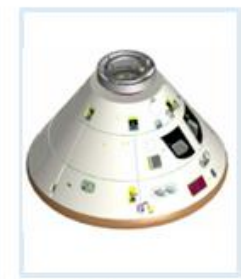

Multi-purpose Crew Vehicle (MPCV)

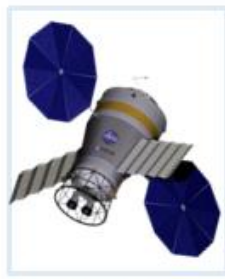

Cryogenic Propulsion Stage (CPS)

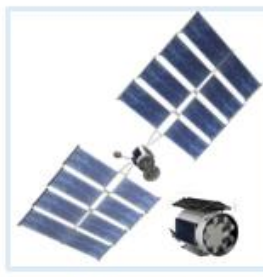

Solar Electric Propulsion (SEP)

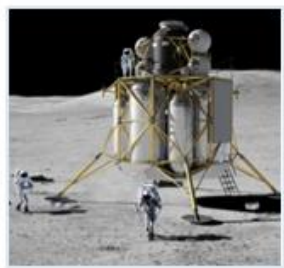

Lunar Lander

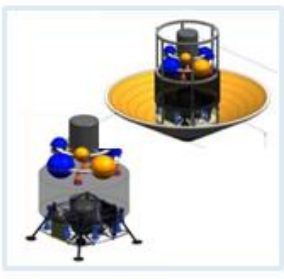

Mars Lander Concepts

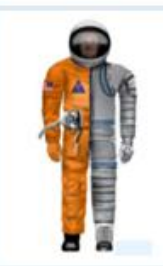

EVA Suit

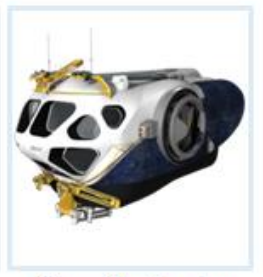

Space Exploration Vehicle (SEV)

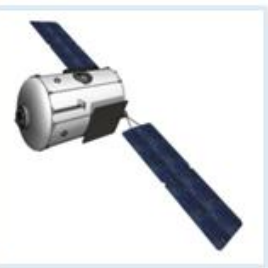

Deep Space Habitat (DSH)

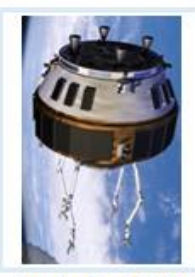

Robotics \& EVA Module (REM)

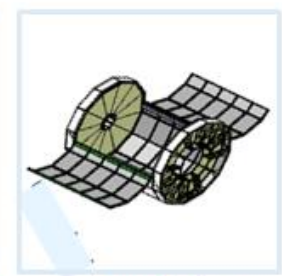

Cargo Hauler

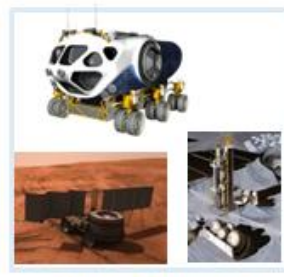

Surface Elements

Figure 2: Notional HAT Architectural Elements 


\section{Technology Needs}

The method used by the HAT team to select the critical technologies is fully documented in [9] and will only be summarized in this paper.

The process began with high level conceptual designs for the architectural elements needed to accomplish the Design Reference Missions (DRMs) and support mission operations at the destinations in Table 1 . The minimum set of adequate technologies to provide the requisite technical capabilities in these architectural elements was determined by consensus of mission planners, spacecraft designers and technology developers.

Cost phasing and "need by" dates (relative to an element's development cycle) were recorded with each technology to assist with HAT's cost estimations for each DRM. Technologies were assumed to be matured and available by the preliminary design review for the enabled element. "Need by" dates for technologies required by multiple elements were based on the element that was expected to be completed first.

Finally, each technology was mapped into the technology classification system [10] used by NASA's Office of the Chief Technologist.

Subject matter experts created technology needs descriptions based on the capability gaps associated with the DRMs and architecture elements, and estimated technology development costs and the fidelity of those costs.

Using this approach, a set of 60 technologies was identified as being critically important for at least one mission under consideration by the HAT ("technology pull"). In addition, "common avionics" was identified as a technology which could substantially improve system level affordability, and four ground operations technologies were identified as having a similar cost reduction potential. The full suite of these 65 technologies is listed in section 5 in Table 2 .

\section{Challenges for JSC Technology Prioritization}

JSC seeks to achieve more effective results from the Center's "seeding" of technology maturation through the Center-level investments in IR\&D and ICA projects. To achieve that objective, it is necessary to create a focus for IR\&D and ICA (technology development) on the set of human spaceflight technology needs that best maps to the JSC strategy. With such a consistent focus and communication of the technology needs JSC has chosen for advancement from year to year, subsequent year's technology projects should build upon previous year's technology project results. Such a focus should also provide an opportunity for the suppliers of the technology development to demonstrate promise for their line of technology advancement, thereby attracting funding from the larger NASA technology funding programs such as the
Office of Chief Technologist's (OCT) Space Technology Program and NASA's Human Exploration and Operations Mission Directorate's Advanced Exploration Systems projects. Using the top-ranked human spaceflight technology needs as identified by the HAT ensures that there is long-term relevance for these IR\&D and ICA technology development projects in advancing the technology to meet a capability or mission needs.

Also to be considered should be the OCT Technology Area Breakdown Structure (TABS), an outline of the OCT Technology Roadmaps. The TABS is effectively a technology discipline view of the key technology advancements needed to enable and enhance NASA's future missions. Advancing a particular technology discipline does not necessarily close a capability gap, especially those requiring advancements from several disciplines. Appendix A reveals that many-to-one and many-to-many relationships exist between many of the OCT TABS and identified HAT technology needs. Also, the technologies in Appendix A suggest many-to-one, many-to-many, and one-to-many relationships to the HAT identified human spaceflight mission architecture elements and missions/destinations listed in Table 1. This means that a single technology may support multiple mission architecture elements, or just one, may support multiple missions, or just one, be addressed by multiple technology areas (or disciplines) in the TABS, or just one, and may support multiple HAT technology needs, or just one. These myriad relationships should have an obvious impact for prioritization of IR\&D and ICA projects to be funded.

Capturing all of the work produced by the HAT, the OCT TABS, the OCT prioritizations of technologies as well as the National Research Council's prioritizations of technologies, and documenting the relationships between them all, as well as with HAT identified human spaceflight mission architecture elements and missions/destinations is not particularly suited to spreadsheet applications. The obvious tool for this was a database, and specifically because of its availability to NASA employees, a Microsoft Access database.

More specifically related to achieving the objective of JSC to achieve more effective results for the Center's funding allocation to IR\&D and ICA projects, the TechNeeds Database required extensions to relate JSC Core Technical Competencies, and the potentials for Partnerships and Commercialization, through an appropriate weighted scoring process.

Section 4 will describe the core of the HAT Technology Needs Database, as well as the extensions that enabled a technology needs to support human spaceflight based prioritization of IR\&D and ICA project funding allocations.

\section{HAT TEChNOLOgY NeEdS DatABASE}

The core data sets included in the TechNeeds Database are described in Figure 3. 


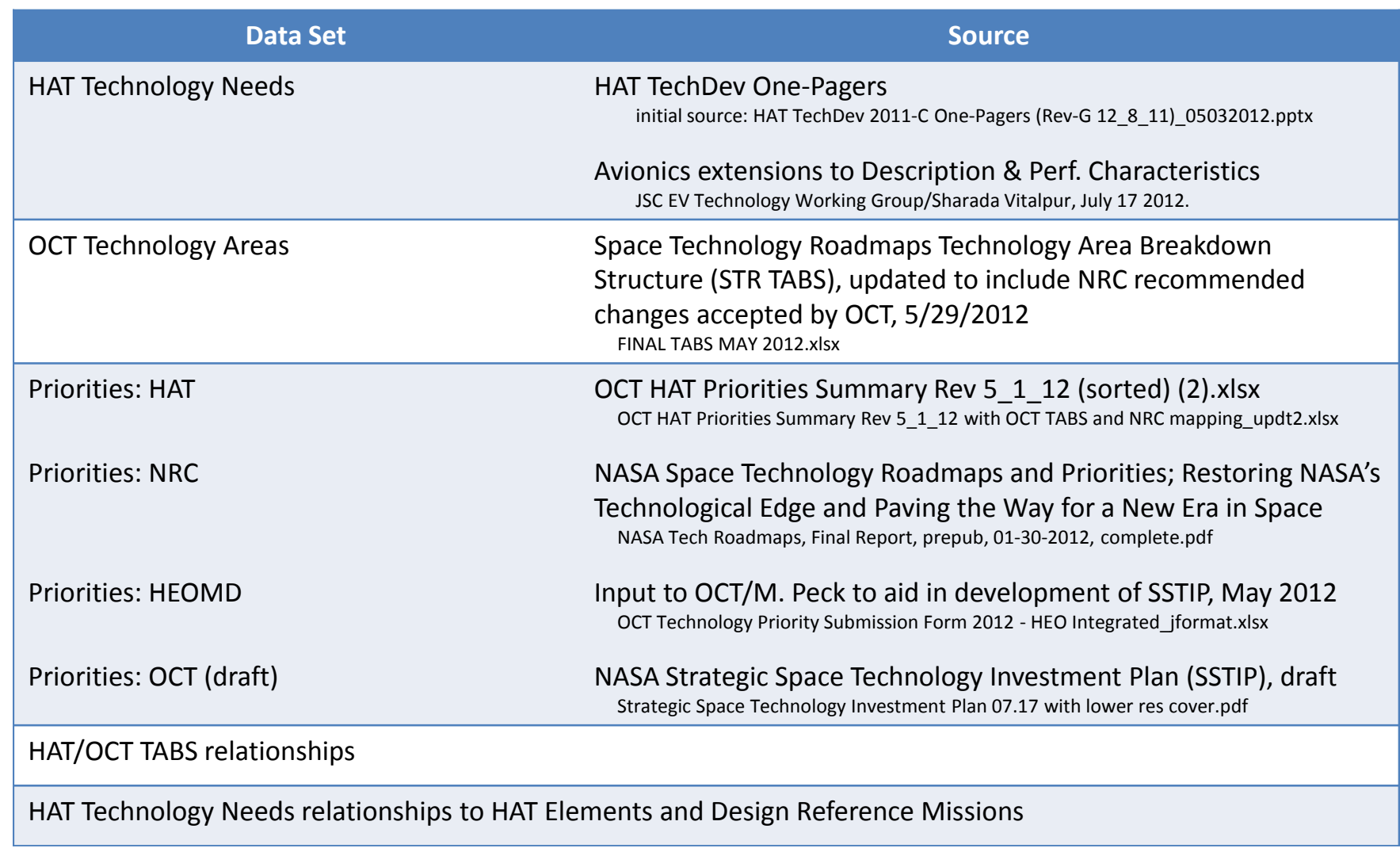

Figure 3: Core Data Sets contained in the HAT Technology Needs Database

The TechNeeds Database has been developed for multiple users. User access can be restricted to selected fields, forms and reports, with read/write and read only capabilities. Several "canned" reports are available from the Main Menu, and new reports can be developed as needed by the user. Many of these "canned" reports were designed to match the format of existing HAT reports that had been generated in Microsoft Excel and PowerPoint, streamlining the update of information while continuing to provide customary reports. After data updates, these canned reports may be generated with the click of a menu button. New data only needs to be entered once. The reports may be printed directly from the database or exported to Microsoft Word, Excel, or PowerPoint formats or to Adobe PDF format. The database provides simple "double-click" navigation between related records.

The JSC extension data sets that have been implemented in the TechNeeds Database are described in Figure 4. Extension data sets do not perturb the core data sets and relationships among them. This ability to extend the TechNeeds Database core for special uses related to human spaceflight technology needs is very powerful. 


\begin{tabular}{|l|l|}
\hline \multicolumn{1}{|c|}{ Data Set } & Source \\
\hline JSC Core Technology Competencies & $\begin{array}{c}\text { Maintained by JSC Chief Technologist Office/AB2 } \\
\text { POC: Steve Prejean }\end{array}$ \\
\hline Partnership Potential - JSC Perspective & $\begin{array}{l}\text { JSC Strategic Opportunities \& Partnership Development Office/AO } \\
\text { POC: Sean Carter }\end{array}$ \\
\hline Commercialization Potential - JSC Perspective & $\begin{array}{c}\text { JSC Strategic Opportunities \& Partnership Development Office/AO } \\
\text { POC: John E. (Jack) James }\end{array}$ \\
\hline Scoring Methods & JSC Chief Technologist Office, JSC Technology Working Group \\
\hline
\end{tabular}

Figure 4: JSC Extension Data Sets contained in the HAT Technology Needs Database

\section{JSC Technology Needs Prioritization}

In the TechNeeds Database, HAT technology needs have been indexed to the NASA OCT Technology Area Breakdown Structure (TABS) to aid in understanding how those capabilities relate to the well-known OCT disciplines.

JSC Core Technology Competencies are based upon input from the Center directorates and indexed by TABS. The competency information is extracted from the $R \& D$ Partnership database [11] maintained by the JSC Chief Technologist Office, and incorporated as a JSC extension into the TechNeeds Database.

JSC, with a strategic goal to "Lead Human Exploration" and a success factor that includes "Lead maturation of human exploration technologies," has a set of core technology competencies that supports the fulfillment of the majority of the Agency's human space flight technology needs (represented by the HAT Technology Needs). JSC's core technology competencies map to 50 of the 65 HAT technology needs as shown in Table 2. 
Table 2. JSC's core technology competencies map to 50 of the 65 HAT technology needs.

OCT Technology Area

01 Launch Propulsion Systems

02 In-Space Propulsion Technologies

Space Power and Energy Storage

04 Robotics, Tele-Robotics and Autonomous Systems

05 Communications and Navigation

06 Human Health, Life Support \& Habitation Systems

07 Human Exploration Destination Systems

09 Entry, Descent and Landing Systems

11 Modeling, Simulation, Information Technology and Processing

12 Materials, Structures, Mechanical Systems and Manufacturing

13 Ground and Launch Systems Processing

14 Thermal Management Systems
HAT Technology Need

JSC Technology

Competency (linked TABS)

Oxygen-Rich Staged Combustion (ORSC) Engine Technology Advanced, Low Cost Engine Technology for HLLV

LOX/Liquid Methane Cryogenic Propulsion System

LOX/Liquid Methane Reaction Control Engines

Non-Toxic Reaction Control Engines

Electric Propulsion \& Power Processing

Nuclear Thermal Propulsion (NTP) Engine

Unsettled Cryo Propellant Transfer

In Space Cryogenic Liquid Acquisition

$300 \mathrm{kWe}$ Fission Power for Electric Propulsion

High Strength/Stiffness Deployable 10-100 kW Class Solar Arrays

Autonomously Deployable $300 \mathrm{~kW}$ In-Space Arrays

Fission Power for Surface Missions

Multi-MWe Nuclear Power for Electric Propulsion

Regenerative Fuel Cells

High Specific Energy Batteries

Long Life Batteries

Precision Landing \& Hazard Avoidance

Telerobotic control of robotic systems with time delay

Robots Working Side-by-Side with Suited Crew

Autonomous Vehicle Systems Management

Automated/Autonomous Rendezvous \& Docking, Proximity Operations, and Target Relative

Crew Autonomy beyond LEO

Common Avionics

High Data Rate Forward Link (Flight) Communications

High Rate, Adaptive, Internetworked Proximity Communications

In-Space Timing and Navigation for Autonomy

Quad Function Hybrid RF/Optical Comm, Optical Ranging, RF Imaging System

Closed-Loop, High Reliability, Life Support Systems

High Reliability Life Support Systems

Deep Space Suit (Block 1)

Lunar Surface Space Suit (Block 2 )

Mars Surface Space Suit (Block 3)

Long Duration Spaceflight Medical Care

Long-Duration Spaceflight Behavioral Health and Performance

Microgravity Biomedical Counter-Measures for Long Duration Spaceflight

Microgravity Biomedical Counter-Measures - Optimized Exercise Equipment

Deep Space Mission Human Factors and Habitability

In-Flight Environmental Monitoring

Fire Prevention, Detection \& Suppression (reduced pressure)

Space Radiation Protection - Galactic Cosmic Rays (GCR)

Space Radiation Protection - Solar Particle Events (SPE)

Space Radiation Shielding - SPE

In-Situ Resource Utilization (ISRU) - Lunar: Oxygen/Water Extraction from Lunar Regolith

In-Situ Resource Utilization (ISRU) - Mars: Oxygen from Atmosphere \& Water Extraction from Soil

Anchoring Techniques \& EVA Tools for u-G Surface Operations

Suit Port

Surface Mobility

Mission Control Automation beyond LEO

Dust Mitigation

Entry, Descent, and Landing (EDL) Technologies - Mars Exploration Class Missions

Entry, Descent, and Landing (EDL) Technologies - Earth Return

Advanced Software Development/Tools

Inflatable: Structures \& Materials for Inflatable Modules

Lightweight and Efficient Structures and Materials

Mechanisms for Long Duration, Deep Space Missions

Low Temperature Mechanisms

Ground Systems: Low Loss Cryogenic Ground Systems Storage and Transfer

Ground Systems: Corrosion Detection \& Control

Ground Systems: Fault Detection, Isolation, and Recovery

Ground Systems: Wiring Fault Detection and Repair

In-Space Cryogenic Propellant Storage (Zero Boil Off LO2; Reduced/Zero Boil Off LH2)

Thermal Control

Robust Ablative Heat Shield (Beyond Lunar Return) - Thermal Protection System

Robust Ablative Heat Shield (Lunar Return) - Thermal Protection Systems

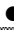

-

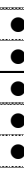

-

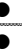

$\bullet$

-

-

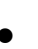

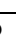

2


The JSC strategy for prioritizing allocation of IR\&D and ICA funding to technology development projects also considered commercial and partnership potential. Commercial and Partnership Potential as implemented in the JSC extensions to the TechNeeds Database is based upon expert knowledge from JSC personnel involved in those areas.

The four criteria for executing this strategy were Human Spaceflight Technology Needs, the JSC Core Technology Competencies, and JSC perspectives for Partnership and Commercialization Potential. The JSC Technology Working Group determined the weights for each of the input criteria. These weights assigned to these criteria appear in Table 3 [12].

Table 3: Prioritization Criteria Weighting

\begin{tabular}{|l|c|}
\hline \multicolumn{1}{|c|}{ Criterion } & Weight \\
\hline Human Space Flight Technology Needs & $40 \%$ \\
\hline JSC Core Technology Competencies & $40 \%$ \\
\hline Commercial Potential - JSC Perspective & $10 \%$ \\
\hline Partnership Potential - JSC Perspective & $10 \%$ \\
\hline
\end{tabular}

JSC experts scored how well each of their designated areas mapped to the objective of their charts.

The Exploration Missions and Systems Office worked with the JSC Chief Technologist's Office and JSC's Strategic Opportunities and Partnership Development Office to assess JSC's IR\&D portfolio and to recommend focus areas for the FY13 and future IR\&D calls. The 27 HAT Technology Needs that have the greatest alignment with the JSC core technology competencies, JSC's partnerships in pursuit, and commercialization potential were selected as topics for the JSC Center-level IR\&D Call for Proposals.

The TechNeeds Database, developed to link HAT Technology Needs to JSC core competencies, partnership potential, and commercialization potential, continues to support the development and execution of a JSC technology strategy. The Strategic Opportunities and Partnership Development Office/AO is using the HAT Technology Needs descriptions and the mapping to the OCT Technology Area Breakdown Structure to identify partnership opportunities between commercial companies and JSC technologists.

\section{Summary}

NASA's Human Spaceflight Architecture Team has identified 65 critical technologies necessary to achieve envisioned future human spaceflight missions. The process used to identify these considered conceptual designs for architecture elements that would support these missions, as well as the OCT list of technology disciplines contained in the OCT TABS. There were many relationships between missions, architecture elements, and NASA priorities that must be considered in developing any cost effective strategy for technology development.

The HAT Technology Needs Database collected the original HAT data and technologies and established all the one-tomany, many-to-one, and many-to-many relationships between all the core data sets and architecture elements and missions that need to be considered in any technology development strategy. The HAT Technology Needs Database provides insights into these critical technology needs that are very difficult to obtain using the flat file spreadsheets of the original HAT core data sets.

JSC used the HAT Technology Needs Database to develop a strategy for IR\&D and ICA funding allocation to technology development projects. This was accomplished by building extension sets to the HAT Technology Needs Database core data sets that reflect JSC Core Technology Competencies, and the potentials for commercialization and partnerships. The HAT Technology Needs Database was further extended by adding JSC prioritization weighting criteria. The results thus enabled more focused proposal calls.

The HAT Technology Needs Database core data sets contain a wealth of information. This information, with the sets of forms and reports built into the HAT Technology Needs Database, can enable NASA organizations interested in satisfying the critical technology needs for future human space mission to use a cost effective approach. Further, the extensibility of the HAT Technology Needs Database allows those organizations to apply their own additional foci, values, and prioritization factors.

To date, the International Space Exploration Coordination Group has added their extension data sets to the HAT Technology Needs Database to identify partnering opportunities for international partner technology area developments. Also, the Homes for Tomorrow group has added an extension to extract technologies that would support dual use of habitation advances for homes on Earth.

To inquire about how your organization can use the HAT Technology Needs Database, contact the author of this paper. 


\section{REFERENCES}

[1] NASA Exploration Systems Mission Directorate presentation, "Human Space Exploration Summary," January 2011.

http://www.nasa.gov/pdf/525162main_HEFT_Final_Brief 508_20110309.pdf [accessed 22 January 2013].

[2]Culbert, C. "Human Space Flight Architecture Team (HAT) Overview," November 2011.

http://www.nasa.gov/pdf/603232main_CulbertHAT\%20Overview\%20for\%20GER\%20Workshop.pdf [accessed 22 January 2013].

[3] Olson, J. "The Way Forward-New Pathways for Human Spaceflight," April 2011.

http://www.nasa.gov/pdf/552846main_New_Pathways_H uman_Spaceflight_Olson.pdf [accessed 22 January 2013].

[4] Olson, J.; Culbert, C.; Laurini, K.C., "NASA's Human Space Exploration Plans and Architecture," 62nd International Astronautical Congress, Cape Town, SA. October 3-7 2011. IAC-11.E3.2.1

[5] Mueller, R.P., Connolly, J.C., Whitley, R.J., "NASA Human Spaceflight Architecture Team: Lunar Surface Exploration Strategies," Global Space Exploration Conference, Washington DC, May 2012. GLEX2012.02.P.17.x12620

[6] Hoffman, Stephen J., Baker, John D., and Voels, Stephen A., "Mars as a Destination in a Capability Driven Framework," ASCE Earth and Space 2012, Pasadena, CA, April 2012.

[7] Friedensen, Victoria, and Mazanek, Daniel D., "NASA's Plans for Human Exploration of Near-Earth Asteroids," ASCE Earth and Space 2012, Pasadena, CA, April 2012.

[8] Bobskill, Marianne R., and Lupisella, Mark L., "The Role of Cis-Lunar Space in Future Global Space Exploration," Global Space Exploration Conference, Washington DC, May 2012. GLEX-2012.05.5.4x12270

[9] Mercer, Carolyn R., Vangen, Scott D., Williams-Byrd, Julie A., Stecklein, Jonette M., Rahman, Shamin A., Rosenthal, Matthew E., Hornyak, David, M., Alexander, Leslie, Korsmeyer, David J., Tu, Eugene L., Alfano, David D., Kumdrot, Craig E., Wiley, Dianne S., Davison, Stephen C., and Balint, Tibor S., "Critical Technology Determination for Future Space Flight," NASA/TM-2012-217670, September 2012
[10] NASA Office of the Chief Technologist, "Space Technology Roadmaps Technology Area Breakdown Structure," May 2012. http://www.nasa.gov/pdf/501627main_STR-IntFoldout rev11-NRCupdated.pdf [accessed 22 January 2013].

[11] NASA, R\&D Partnership database maintained by the JSC Chief Technologist Office.

https://rdpartnership.jsc.nasa.gov/Report.cfm?Center=JSC [accessed 17 January 2013].

[12] JSC Chief Technologist Office, “JSC Technology TABS Roadmap Charts," December 2012.

http://www.nasa.gov/centers/johnson/pdf/716017main T ABS JSC_STR_Packet_12_20_2012A.pdf [accessed 22 January 2013].

\section{BIOGRAPHY}

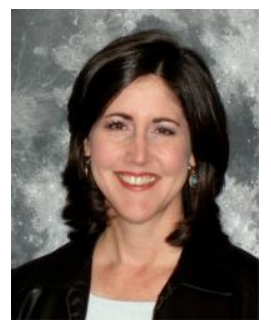

Jonette M. Stecklein leads the Technology Development and Integration activities for the Exploration Mission and Systems Office, defining and prioritizing the technology advancements needed to better implement missions beyond low Earth orbit and enabling humans to live and work in space. Previously, Ms. Stecklein led the technology development activities for the Lunar Surface Systems Project Office in NASA's Constellation Program. As a member of the NASA International Space Station (ISS) team for twelve years, Ms. Stecklein led efforts within all phases of the ISS Program lifecycle, including Requirements Team Manager (responsible for the development of the ISS System, Segment, and End Item Specifications). as Physical Integration Lead (verifying that inter-element structure, cables, and fluid lines would mate successfully during ISS on-orbit assembly), and Crew Health Care Systems Integrator (defining the customer requirements and acquiring hardware/software systems to be integrated and operated on-board ISS). Prior to joining the ISS Program, Ms. Stecklein worked in $J S C$ 's Engineering Directorate as the Chief Engineer and Lead Systems Engineer for the Artemis Common Lunar Lander project, an in-house development of a robotic lunar lander. Ms. Stecklein holds a BS in Aerospace Engineering and an MS in Systems Engineering. She served on the Board of Directors for the International Council on Systems Engineering (INCOSE) in 2008 and 2009.

\section{ACKNOWLEDGEMENTS}

The TechNeeds Database would not exist without the efforts of Alida Andrews, who spent many hours constructing the database, the forms, and reports that are built in as tools. 


\section{APPENDIX A: HAT TECHNOLOGY NEEDS MATCHED TO JSC TECHNOLOGY COMPETENCIES}

The following list summarizes the description and performance characteristics for each of the 50 technology needs that are supported by JSC core technology competencies (as noted in Table 2). Acronyms are spelled out in Appendix B. The complete suite of 65 critical technologies identified by the HAT team is described in detail in [9].

The technologies are grouped according to the Technology Area Breakdown Structure published by NASA's Office of the Chief Technologist [10]. In some cases a single HAT technology maps to multiple OCT technical areas. Technology Area TA 08 Science, Instruments, Observatories and Sensor Systems, and TA 10 Nanotechnology, are not used by the HAT.

Note that this summary is a work in progress, and evolves commensurate with updates in scope and level of detail of the HAT DRMs. Differences exist in the level of detail provided below. Some of these differences are caused by differing levels of fidelity currently existing between the reference missions; others result from the differing TRL levels of the technology.

This data is based on information collected during 2011 Cycle-C of the HAT process, and is current as of December 8, 2011.

The following information fields are provided.

- Title: Brief descriptive title of the technology development

- Discipline: HAT categorization of the technology (e.g., Chemical Propulsion, Advanced Propulsion, etc.)

- OCT TA\#: Cross reference to the NASA OCT Technology Area Breakdown Structure

- Description: Explanation of how and why a specific technology development is required

- Performance characteristics: Identifies the advancements needed over current state-of-the-art. Provides targets for technologists to work towards to close the capability gap and enable the linked design reference missions.

\section{TA 01 LAUNCH PROPULSION}

Enhance existing solid or liquid propulsion technologies by lower development and operations costs, improved performance, availability and increased capability.

HAT Technology Needs in this technology area do not map to JSC Core Technology Competencies. See [9] for details of HAT technologies in this discipline area.
TA 02 IN-SPACE PROPULSION

Advancements in conventional and exotic propulsion systems, improving thrust performance levels, increased payload mass, increased reliability, and lowering mass, volume, operational costs, and system complexity.

\section{Liquid Oxygen/Liquid Methane Cryogenic Propulsion System}

Chemical Propulsion (OCT TA 2.1)

Description

- An In-Space Stage, powered by a demonstrated workhorse engine, intended for mission applications beyond LEO.

- The oxygen and methane propellant combination has the potential for good engine performance, which can result in lower vehicle mass and greater payloadcarrying capability.

Performance characteristics

- Improved handling and non-toxicity benefit of the LCH4/oxygen combination (hours rather than days ground operations)

- Approximately $10 \%$ specific impulse performance improvement relative to hypergolic systems.

\section{Liquid Oxygen/Liquid Methane Reaction Control Engines}

Chemical Propulsion (OCT TA 2.1)

Description

- The oxygen and methane (LCH4) propellant combination has the potential for greater engine performance, which can result in lower vehicle mass and greater payload-carrying capability.

- Demonstrated performance of a TRL 6 engine including:

- Specific impulse of 317-s; Impulse bit of $4 \mathrm{lbf}-\mathrm{s}$; 50,000 cycles with a cryogenic valve;

- Ignition and operation over a range of propellant inlet conditions (liquid/liquid to gas/gas)

Performance characteristics

- Improved handling and non-toxicity benefit of the LCH4/oxygen combination (hours rather than days ground operations).

- Approximately $10 \%$ specific impulse performance improvement relative to hypergolic systems.

Non-Toxic Reaction Control Engines

Chemical Propulsion (OCT TA 2.1)

Description

- Propulsion system technologies for non-toxic or "green" propellants for use in reaction control systems.

- Non-toxic technologies for RCS engines over the thrust range of 25 to 1000 lbf. Propellant options include hypergolic ionic liquids and nitrous oxides monopropellants, both of which can be easily stored in space and on the ground.

Performance characteristics 
- Improved handling and non-toxicity benefit of hours rather than days ground operations.

- Non-toxic bipropellant or monopropellants that have higher specific impulse (greater than hypergolic) and/or high specific impulse density (greater than hypergolic) with better safety and reduced handling risks

\section{Unsettled Cryogenic Propellant Transfer}

\section{Cryogenic Fluid Systems (OCT TA 2.4)}

Description

- Efficient transfer of cryogenic fluids in-space is required for propellant resupply to a Cryogenic Propulsion Stage (CPS) and/or oxygen resupply to a Deep Space Habitat (DSH) and has direct planetary application to ISRU Surface Systems. The SOA for propellant transfer in cryogenic upper stages requires the use of an ancillary propulsion system to settle the cryogenic propellants at the tank outlets and a helium pressurant system to maintain a constant tank pressure (LO2 only) during propellant transfer. After engine start up the thrust generated by the propulsion system maintains the propellants at the tank outlet and the LH2 tank uses an autogenous gaseous hydrogen pressurant system. This is not possible for tank-to-tank transfers; "unsettled" transfer is also beneficial for propellant resupply of large tank-to-propulsion systems.

- A pumped transfer at unsettled conditions and without a liquid acquisition device in the storage requires a 2-phase fluid tolerant pump for liquid transfer. A transfer process requires a robust leak-free fluid transfer coupling to mate the storage tank and the propulsion system receiver tank, an efficient transfer line chill down technique to minimize the liquid used to chill down the transfer line and a micro-g gauging concept to verify the high fill fraction of the propulsion system receiver tank. An automated propellant leak detection system would ensure the safe in-space transfer operation.

- These technologies are also directly applicable to LO2/Methane propellant systems.

In-Space Performance Characteristics (<0.00003 g).

- 2 Phase Fluid Tolerant Transfer pump: operation to a vapor fraction of $\sim 0.8$ with cryogenic fluids

- Automated Fluid Coupling: leakage < 10-3 sccs gHe after 1000 cycles

- Leak Detection: TBD

- Mass gauging: < $2 \%$ uncertainty of measurement

- Fill Fraction of propulsion system receiver tank: $>0.9$

- Minimum Fluid used to chill transfer lines: $<1 \%$ of transfer line mass

\section{In Space Cryogenic Liquid Acquisition}

\section{Cryogenic Fluid Systems (OCT TA 2.4)}

Description

- Cryogenic liquid acquisition technology is needed for 1) unsettled tank-to-tank propellant transfer, 2) unsettled tank-to-engine propellant transfer, and 3) propellant transfer into heat exchangers needed to maintain propellant tanks at required temperature and pressure. It is important to transfer only cryogenic liquids for these applications, without transferring ullage gas. Propulsive maneuvers can be used to settle the cryogens to ensure liquid-only transfer, but this parasitic propellant burn increases system mass, particularly for the frequent transfers needed for the thermodynamic vent system for tank pressure and temperature control.

- In micro- and reduced-gravity, liquid tends to cling to the walls of the tank, making it difficult to sufficiently cover the tank outlet during fluid outflow.

- An in-space liquid acquisition device (LAD) is required to acquire vapor-free liquid from a propellant tank in micro-g. LADs represent the first stage in successful fluid transfer from a tank to a propulsion system (or another tank). LADs rely on surface tension forces to separate liquid and vapor in the tank and capillary flow to maintain communication between liquid and the outlet during expulsion.

- A second system required for in-space liquid acquisition for large propellant storage and long duration missions is an autogenous pressurant system. Helium pressurant supply is impractical for these missions due to the helium mass required and the large launch mass penalty. An alternative to helium pressurization would be to extract a small amount of liquid or two phase fluid and feed it though a heat exchanger to vaporize the liquid and return it to the tank as a pressurant.

- These technologies are directly applicable to LO2/Methane propellant systems.

- LADs have a proven flight heritage when using higher surface tension storable liquids (e.g., hydrazine), but have not yet been tested in cryogenic liquids $(\mathrm{H}$ and O) in low-g environments.

In-Space Performance Characteristics ( $<0.00003 \mathrm{~g}$ )

- Ratio of LAD delivery system pressure drop to BPP drop at maximum outflow rate $-<0.75$ to 0.5

- Percent of LAD residual LH2 mass to total tank LH2 mass (Expulsion efficiency) - < 1 to $3 \%$

- Ratio of total autogenous pressurant system mass to the mass of equivalent helium pressurant system - < 0.8 to 1.0

\section{TA 03 SPACE POWER AND ENERGY STORAGE}

Improvements to lower mass and volume, improve efficiency, enable wide temperature operational range and extreme radiation environment over current state-of-the-art space photovoltaic systems, fuel cells, and other electrical energy generation, distribution, and storage technologies.

\section{0 kWe Fission Power for Electric Propulsion}

\section{Power Systems (OCT TA 3.1) \\ Description}

- Fission power systems being developed for surface applications can be used to power electric propulsion vehicles.

Performance characteristics 
- Moderate power, low mass $(<30 \mathrm{~kg} / \mathrm{kWe})$ power system for Nuclear Electric Propulsion

- $1200 \mathrm{~K}$ Li-cooled unfueled reactor, 2 x $340 \mathrm{kWe}$ Brayton power conversion, $500 \mathrm{~V}$ power management and distribution

High Strength/Stiffness Deployable 10 to $100 \mathrm{~kW}$ Class Solar Arrays

Power Systems (OCT TA 3.1)

Description

- High power, high voltage, autonomously deployable surface solar arrays in $1 / 6^{\text {th }}$ to $1 / 3^{\text {rd }}$ gravity environments are needed to generate reliable electric power for surface outpost elements over the mission duration. In addition, applications for in-space use with flight elements requires operations during low-g accelerations under propulsion $(0.1 \mathrm{~g})$. Enabling features include compact stowage, reliable deployment in partial gravity, on an irregular surface and dusty environment, Martian wind load strength, EVA compatibility, dust mitigation to limit photovoltaic power degradation and robust to surface arcing environment (Martian surface triboelectric charging). Few options exist and only at the conceptual level. These include mast deployed vertical, Sun-tracking blanket solar arrays for lunar polar surface mission and horizontally deployed, fixed tent like solar arrays. Solar array panels would employ low mass, flexible panel substrates populated with advanced photovoltaic cells, like inverted metamorphic triple junction solar cells, with bandgap tuning for the Martian surface solar spectrum. substrates.

- These solar arrays would power outpost surface elements (e.g., habs/labs, rovers, ISRU, lander/ascent stages, etc.)

- These solar arrays would power in-flight space elements (e.g., CPS, DSH)

Performance characteristics

- High power (10-100 kW),

- High voltage $(<\sim 200 \mathrm{~V})$

- Autonomously deployable surface solar arrays in $1 / 6^{\text {th }}$ to $1 / 3^{\text {rd }}$ gravity environments

- Operational under low-g propulsion accelerations (0.1g)

\section{Autonomously Deployable $300 \mathrm{~kW}$ In-Space Solar Arrays}

Power Systems (OCT TA 3.1)

Description

- High power, high voltage, autonomously deployable solar arrays are required to generate reliable electric power for the SEP Stage over its mission duration. Enabling features include compact stowage, reliable deployment, $\sim 0.1-\mathrm{g}$ deployed strength and robust performance through the mission end-of-life. Leading options include large, dual-wing structures $(2 \times 200$ $\mathrm{kW})$ and modular, sub-wing structures $(20 \times 20 \mathrm{~kW})$ employing advanced photovoltaic cells on flexible substrates. Fine pointing requirements for concentrator-based arrays may limit functionality for some missions, so both planar and concentrator architectures should be considered.

\section{Performance characteristics}

- High power ( 400 kW at beginning of life)

- High voltage $(\sim 350 \mathrm{~V})$

- Low mass and low stowed volume (TBD W/kg and $\left.\mathrm{W} / \mathrm{m}^{3}\right)$

- Cost (2X reduction)

Multi-MWe Nuclear Power System for Electric Propulsion

Power Systems (OCT TA 3.1)

Description

- Nuclear power system development for very high power electric propulsion vehicles to deliver cargo and/or crew to Mars. Once built, this system would also reduce the cost of transits to the Moon, E-M L1, NEOs, and the Martian moons.

Performance characteristics

- High (>1 MWe) power, low mass $(<15 \mathrm{~kg} / \mathrm{kWe})$ power system for nuclear electric propulsion.

- Flight power system development and qualification

\section{Regenerative Fuel Cells}

Power Systems (OCT TA 3.2)

Description

- Long duration energy storage is required for extended surface missions to store solar energy and provide power during low insolation. Applicable to Lunar or Mars surface applications requiring high power and/or long sortie durations.

- RFC system includes a fuel cell and an electrolyzer, each of which can be used independently for power/water generation and $\mathrm{H} 2 / \mathrm{O} 2$ generation, respectively. Electrical power can be used for any vehicle. Water and $\mathrm{O} 2$ can be used for life support for crewed vehicles. Also applicable to ISRU.

- Technology development includes reducing the number of ancillary components to increase reliability and operational lifetime, and reduce parasitic power losses, mass, and volume.

Performance characteristics

- Power generation $>10 \mathrm{kWe}$ for $8 \mathrm{hr}$ or more

- Operable with reactants at $>2000$ psi to reduce tank volume

- Round trip energy conversion efficiency $>50 \%$

- Minimize mass (TBD Wh/kg)

- Operational life $>10,000 \mathrm{hr}$

\section{High Specific Energy Batteries}

Power Systems (OCT TA 3.2)

Description

- Batteries with very high specific energy and energy density are required to enable untethered EVA missions lasting $8 \mathrm{hr}$ within strict mass and volume limitations. Batteries are expected to provide 
sufficient power for life support and communications systems, and tools including video and lighting. Advanced batteries are enhancing for every other vehicle.

Performance characteristics

- Battery-level specific energy > $325 \mathrm{Wh} / \mathrm{kg}$ and energy density $>540 \mathrm{Wh} /$ liter

- $8 \mathrm{hr}$ operation per mission over an operating temperature of 10 to $30^{\circ} \mathrm{C}$.

- Nominally 100 cycles and 5 yr calendar life

\section{Long Life Batteries}

Power Systems (OCT TA 3.2)

Description

- Long life and low temperature survivable batteries will enable Lunar night survival and operations. Polar Craters Ops will require batteries that can survive a cryogenic thermal environment.

Performance characteristics

- Battery-level specific energy > $220 \mathrm{Wh} / \mathrm{kg}$ and energy density > $410 \mathrm{Wh} /$ liter at a $\mathrm{C} / 10$ discharge rate

- Operate at lunar night temperatures for $14 \mathrm{~d}$

- Operate in a perennially shadowed region such as a polar crater

TA 04 ROBOTICS, TELE-ROBOTICS, AND

\section{AUTONOMOUS SYSTEMS}

Improvements in mobility, sensing and perception, manipulation, human-system interfaces, system autonomy are needed. Advancing and standardizing interfaces for autonomous rendezvous and docking capabilities will also be necessary to facilitate complex in-space assembly tasks.

\section{Precision Landing and Hazard Avoidance}

EDL (OCT TA 4.1, 4.5)

Description

- Need autonomous landing and hazard avoidance systems, including terrain relative navigation, that operate in all lighting conditions, including darkness. Autonomous Landing and Hazard Avoidance Technology would enable a first of a kind development for planetary precision landing and hazard avoidance.

\section{Performance characteristics}

- The components and techniques have been simulated and tested to TRL 5 but a full set of integrated field test is needed to show TRL 6 and applicability to future missions

- Need 90-m accuracy at 3- $\sigma$ uncertainty relative to premission identified landing location. Need $0.3 \mathrm{~m}$ hazard recognition and avoidance.

Telerobotic Control of Robotic Systems with Time Delay

Robotics and Mobility (OCT TA 4.3)

Description

- Enable astronauts in vehicle, habitat, or EVA to remotely operate robots at destinations (natural environment and variable time-delay) to collect samples, deploy instruments, etc.
- IVA SOA = control of robot arm in structured environment with man-made payloads and zerodelay (e.g., ISS crew uses SSRMS to move/position cargo modules).

- EVA SOA = none (no EVA control of external space robots exists).

- Enable Earth ground control to remotely operate robots in dynamic environments beyond LEO to support crew (e.g., reconnaissance, survey, site prep, follow-up, etc. during sleep periods)

- Ground control SOA = Single command sequence per day of slow ground robot in static environment without humans (e.g., Mars Exploration Rovers driving few $\mathrm{m} /$ day)

- Enable use of robots deployed by precursor mission, race-ahead or crew in mixed ops modes: beforesupporting - after crew, ground control and crew, IVA and EVA

Performance characteristics

- IVA: Advance SOA to enable telerobotics from inside crew vehicle (e.g., approach/orbit NEO)

- Robot functions: detail reconnaissance, sample collection, worksite prep, etc.

○ Time-delay: $5 \mathrm{~s}$ (orbit-to-surface) to $5 \mathrm{~min}$ (for race-ahead mission architectures)

- EVA: Advance SOA to enable telerobotics from suited crew (in-space or on-surface)

- Robot functions: mobile camera, materials/payload transport, etc.

- Time-delay: up to $10 \mathrm{~s}$

- Ground control: Advance SOA to enable telerobotics in dynamic environments (e.g., tumbling NEO)

○ Robot functions: initial reconnaissance, systematic survey, site prep, follow-up, etc.

o Time-delay: up to $40 \mathrm{~min}$ (Earth to Mars orbit round-trip)

\section{Autonomous Vehicle Systems Management}

\section{Avionics and Software (OCT TA 4.5)}

Description

- Enables autonomous vehicle management with limited crew effort and little to no ground oversight. This autonomous capability is required to ensure safe vehicle operations and monitoring of complex systems, especially at increased distances from Earth where communications time delays are present.

Performance characteristics

- Enable on-board vehicle systems management for mission critical functions at destinations with $>3 \mathrm{~s}$ time delay

- Enable autonomous nominal operations and FDIR for crewed and uncrewed systems

- Reduce on-board crew time to sustain and manage vehicle by factor of $2 \mathrm{x}$ at destinations with $>6 \mathrm{~s}$ time delay (see "Crew Autonomy" description)

- Reduce Earth-based mission ops "back room engineering" requirements for distant mission support delay (see "Mission Control Automation" description) 


\section{Common Avionics}

Avionics and Software (OCT TA 4.5)

Description

- Develop common avionics components such as flight computers, sensors, high performance, environmentally tolerant, interoperable computing and data busses which can be utilized by multiple vehicles. This approach provides support for:

- Multiple architectures to enable single spares to fulfill multiple electronic functions,

- Adaptability to system failures,

- Redundancy by providing adaptable spares, and

- Multiple interconnection options.

Performance characteristics

- Exceed $75 \%$ commonality of avionics components across HAT DRM elements for reusability (on-orbit spares) and supportability

- Enable up to 1/3 of Planning and Analysis software tools (used in MCC "backroom" today) to be run onboard the vehicle

- Reduce power use by $30 \%$ for same processing power

- Reduce avionics weight by $50 \%$ for same processing power

- Improve reliability of avionics components, thereby improving crew safety and reducing logistics mass

Automated/Autonomous Rendezvous and Docking, Proximity Operations and Target Relative Navigation

$A R \& D(O C T T A$ 4.6, 4.2, 4.5)

Description

- Maturation of subsystem technologies (relative navigation sensors, GN\&C flight software, system managers, and mechanisms) to accomplish autonomous rendezvous and proximity operations for various in-space destinations such as satellite servicing and NEA exploration. The benefit of this technology development is to improve human safety, improve mission performance and flexibility by enabling autonomous rendezvous and proximity operations interactions with complex or uncontrolled planetary bodies.

\section{Performance characteristics}

- System performance driven by the need for autonomous operations; high reliability, rapid missionization, rendezvous with non-cooperative targets with unknown geometry, tumbling attitude, and unknown surface features; and mass/power constraints. Rendezvous missions include flybys of destinations without landing or docking. Proximity operations require loiter at destinations with zero relative velocity. Major challenges include the ability to rendezvous and dock in all ranges of lighting, work across near to far range, and achieve a docked state in all cases.

\section{Crew Autonomy Beyond LEO}

Avionics and Software (OCT TA 4.7, TA 6)

Description
- Autonomous Crew Operations (planning, commanding, fault recovery, maintenance) in Beyond LEO missions. Systems and Tools to provide the crew with independence from Earth-based ground operations support. Such crew autonomy is essential to accommodate the ground communication delays and blackouts at distant locations.

Performance characteristics

- Enable crew nominal operation of vehicle or habitat at destinations with $>6 \mathrm{~s}$ time delay to ground

- Enable coordinated ground and crew nominal operations at destinations with $>6 \mathrm{~s}$ time delay (See "Mission Control Automation" description)

- Enable crew to detect off nominal situations and put vehicle in safe configuration without ground coordination

\section{Robots Working Side-by-Side with Suited Crew}

Robotics and Mobility (OCT TA 4.7, 4.4)

Description

- Human mission activities can be performed more effectively if robotically assisted. Coordinated efforts between humans and machines/robots can improve the mission risk/productivity trade space.

- The top technical challenges in human-robot interactions are multi-sensor feedback, understanding and expressing intent between humans and robots, and supervised autonomy of dynamic/contact tasks.

- When robots and humans need to work in close proximity, sensing, planning, and autonomous control system for the robots, and overall operational procedures for robots and humans, will have to be designed to ensure human safety around robots.

- The goal is to enable EVA crew and machine interaction without real-time control and support needed from IVA or ground control personnel.

Performance Characteristics

- Avoid need for IV robot controller Avoid need for IV spotter/checker Avoid dependence on Mission Control

- Create force level safety for proximity operations.

- Create multi-modal human-robot interfaces and autonomy software.

- Create fault tolerant free flyer and EVA positioning technology.

- Create asteroid sampling, processing, manipulation.

- Create asteroid grappling and anchoring technology.

\section{TA 05 COMMUNICATION AND NAVIGATION}

Technology advancements to enable higher forward and return link communication data rates, improved navigation precision, minimizing latency, reduced mass, power, volume and life-cycle costs.

High Data Rate Forward Link (Flight) Communications Communications (OCT TA 5.2)

Description

- Combine transmitters on the ground across an array of antennas to produce uplink data rates 3-4 orders of 
magnitude higher performance than current DSN capabilities.

- Supports uplinked video, imagery and software uploads. Enables spacecraft receiver to receive high data rate with reduction avionics size, weight and power burden to Elements. Leverages navigation improvements in orbit determination accuracy and trajectory management from improved communication link.

\section{Performance characteristics}

- Enable uplink rates: 25-50 Mbps at $1 \mathrm{AU}$ using Xband

- Size and weight reduction: compared to currently achievable receiver: $>50 \%$

- Leverage navigation improvements in orbit determination accuracy and trajectory management from improved communication link

\section{High-Rate, Adaptive, Internetworked Proximity Communications}

\section{Communications (OCT TA 5.4)}

Description

- Enable high data rate communications between multiple in-space elements for situational awareness, enable element proximity radios to sense RF conditions and adapt autonomously, enable elements to store, forward, and relay/route information to other elements intelligently and when communications is available, enable element radios to be reprogrammed from ground based on in-situ characterization of the NEO environment. The benefit of this technology development is to improve situational awareness and communications, improving operational efficiency.

\section{Performance characteristics}

- Data rate: $>20 \mathrm{Mb} / \mathrm{s}$ simultaneously between peers

- Employ multiple frequency/modulation/coding/ power schemes, including low frequency schemes to enable low rate, non-line of sight communication through small NEO's when relay through other elements is not available. (Max range: < $20 \mathrm{~km}$. Max NEO size for penetration: $<50 \mathrm{~m}$ )

- Max storage time: $<5 \mathrm{~min} /$ Element at $20 \mathrm{Mb} / \mathrm{s}$

- Max routing: <20 destinations and/or elements

- Enable radios to be adapted in frequency of operation, modulation and coding to information as it is discovered about the NEO environment in near realtime. (Near real-time: < 30 min of each NEO Quad Function Hybrid RF/Optical Comm, Optical Ranging, RF Imaging System

\section{Communications (OCT TA 5.5)}

Description

- This technology provides the capability to perform four functions with a single system: RF and optical communication, optical ranging and RF imaging. This enables:

- Reduced avionics size, weight and power burden to Elements through combined RF/Optical capability in a single system.
- Multiple elements to aggregate communications through a single element to solve spectrum and 'multiple spacecraft located in the same aperture' issues on the Earth side.

- Reliable high data rate communications between in-space elements and ground regardless of distance from Earth and availability of assets on the ground-side, to conserve element power whenever possible,

- Simplified tracking of terminal by providing simultaneous RF beacon capability with terminal while optical system is operating.

- This is a recommended technology for missions where both imaging and long-range, high rate communications are required for the mission.

\section{Performance characteristics}

- Power savings during optical mode: < TBD W. Size and weight reduction compared to dual systems: $<40$ $\%$

- Optical data rate to $0.5 \mathrm{AU}$ from Earth: $>1 \mathrm{~Gb} / \mathrm{s}$ simultaneous uplink and downlink with ground

- NEO's/NEA's at 0.5 AU distance or greater, including Mars missions

\section{TA 06 HUMAN HEALTH, LIFE SUPPORT, AND}

\section{HABITATION SYSTEMS}

Improvements in reliability, maintainability, reduced mass and volume, advancements in biomedical countermeasures, and self-sufficiency with minimal logistics needs are essential for long duration spaceflight missions. In addition, advancements in space radiation research are required, including advanced detection and shielding technologies.

\section{Closed-Loop, High Reliability, Life Support Systems}

\section{Life Support (OCT TA 6.1)}

\section{Description}

- Enhance and develop new, flexible Environmental Control and Life Support (ECLS) process technologies and systems to reliably increase system closure and reduce logistics, enabling autonomous long duration human exploration missions.

- Based on systems analysis and trade studies, targeted functions and technologies may include:

- Close the Atmosphere Revitalization (AR) loop by furthering $\mathrm{O}_{2}$ recovery, and reducing logistics. Technologies may include Bosch, methane processing, and solid oxide electrolysis as well as advanced trace contaminant control and filtration.

- Further closure of the Water Recovery (WR) loop by processing brines. Reduce clothing logistics and enhance crew health by enabling water recovery from laundry and hygiene wastewaters, respectively. May also include purification of water derived from ISRU sources.

- Processing of solid waste to recover water, reduce volume, and stabilize for long-term storage. Technologies include compaction, drying and mineralization of solid wastes, including trash, 
feces and solid byproducts from AR and WR processes.

- Opportunities to develop common technologies, processes, and components suitable for multiple vehicle and mission applications can enhance the overall sustainability of human space exploration.

- Bring technologies to TRL 6 through progressive levels of ground-based integrated testing and ISS flight demonstrations. Perform long duration human in the loop testing to flush out hardware closed-loop issues such as contaminant buildup.

- NOTE: "High Reliability Life Support Systems" is a subset of this technology item.

Performance Characteristics

- Approach $100 \%$ closure for water and oxygen. Enable vehicle and mission autonomy through high reliability, significantly reduced consumable mass, and reduced dependency on logistics.

- Meet new vehicle requirements including operation in more extreme cabin environments (reduced pressure [8 psia] and elevated $\left.\mathrm{O}_{2}[\approx 32 \%]\right)$, reclamation of more complex process streams, and planetary protection.

\section{High Reliability Life Support Systems}

Life Support (OCT TA 6.1)

Description

- Development and validation of open and closed-loop Environmental Control and Life Support Systems (ECLSS), including Atmosphere Revitalization, Water Recovery, Waste Management and Crew Accommodations, focused at improving reliability and reducing logistics over the state of the art.

- Base technology selection and development on systems analysis and trade studies. Deliver new gapfilling technologies identified by vehicle elements including common adjustable pressure regulator capable of controlling a range of cabin, suit loop, and EVA suit pressures, low maintenance human waste collector and trash compactor, clothing, washer and dryer.

- Bring technologies to TRL 6 through progressive levels of ground-based integrated testing and targeted flight demonstrations for selected process technologies. Perform long duration testing to address hardware reliability issues.

- Opportunities to develop common technologies, processes, and components suitable for multiple vehicle and mission applications can enhance the overall sustainability of human space exploration

\section{Performance Characteristics}

- Meet or exceed performance over current state of the practice $(\approx 90 \%$ recovery of water from urine and humidity condensate, and $\approx 50 \%$ of $\mathrm{O}_{2}$ from $\mathrm{CO}_{2}$ ).

- Meet new vehicle element requirements:

○ More robust and reliable common components (e.g., fans, separators, pumps, sensors) to support longer (unmanned) loiter and extended mission durations that withstand the launch/landing loads environments and thermal/dust environments.

- Increased vehicle autonomy, including high reliability, reduced logistics and in-flight reparability;

- More extreme cabin environmental conditions (reduced pressure [8 psia] and elevated $\mathrm{O}_{2}[\approx 32 \%]$ )

- More complex process streams for recycling (wastewater from trash, hygiene and laundry).

\section{Deep Space Suit (Block 1)}

EVA (OCT TA 6.2)

Description

- EVA suit with rear entry capability and crew-cabin pressure matching for compatibility with Suit Port; improved life support systems for increased life, reliability, and flexibility; and improved poweravionics-software to increase crew autonomy and work efficiency.

Performance characteristics

- Suit - rear entry suit, capable of operations at $\sim 8$ psid (SOA is 4.3 psid)

- DSH needs: Dexterous gloves for IVA contingency repairs while the cabin is depressurized.

- Experience shows that EVA repair inside a cabin is not practical (suits are too bulky), but IVA suited repair may be possible, if gloves are flexible enough for fine motor skill work.

- Portable Life Support System (PLSS)

- Variable set point oxygen regulator provides more flexibility for interfacing with multiple vehicles, the ability to start an EVA at an 8 psid pressure driven by a suit port and then decrease pressure mid-EVA for improved mobility, and treat decompression sickness in the suit (variable between 0 and 9 psid)

- On-back regenerable $\mathrm{CO} 2$ and humidity control (eliminates consumables)

- Robust water loop that can handle low quality water, long duration missions, low pressure operations, and bubbles (> 50 EVA life)

- Power-Avionics-Software (PAS)

- Compatible with high specific energy battery (> $235 \mathrm{~kW}-\mathrm{hr} / \mathrm{kg}$ )

- Radio that is network capable for missions involving multiple assets (vehicles and suits) and has data rates that support transmitting high definition video (> $10 \mathrm{Mbps}$ )

- EVA display (either helmet mounted or handheld) that improves upon the 12 character LCD and laminated flip cards used on ISS

- EVA information system that increases crew autonomy and work efficiency

Lunar Surface Space Suit (Block 2)

EVA (OCT TA 6.2)

Description 
- Suit Port-compatible EVA suit for surface destinations with small gravity field and hard vacuum atmosphere (e.g., Lunar surface)

\section{Performance characteristics}

- Assumptions:

- Block 2 development occurs after Block 1 (deep space suit). Block 1 development is successful and technologies can be transferred to Block 2 as appropriate

- Pressurized rover concept of operations with suit port

- Lunar surface or other mission with small gravity field and hard vacuum atmosphere

- For example, a Mars mission with $1 / 3 \mathrm{~g}$ and low pressure $\mathrm{CO} 2$ atmosphere would require additional development due to environmental constraints

- Technical changes from Block 1 to Block 2

- Suit: improved lower torso mobility

- Portable Life Support System (PLSS): upgrade to dust tolerant components (quick disconnects, relief valves, etc...)

- Power-Avionics-Software: upgrade to dust tolerant electrical connectors, switches, and controls; increase the capabilities of the information system for additional autonomy; take advantage of advances in battery or avionics components as appropriate

\section{Mars Surface Space Suit (Block 3)}

\section{EVA (OCT TA 6.2)}

Description

- Suit Port-compatible EVA suit for surface destinations with intermediate gravity field $(1 / 3 \mathrm{~g})$ and low pressure atmosphere (Mars)

\section{Performance characteristics}

- Assumes Block 3 development occurs after Block 1 (deep space suit) and Block 2 (surface suit for moons).

- Technical changes from Block 2 to Block 3

- All EVA systems components have an increased need for decreased mass

- Suit: additional emphasis on boots, thermal insulation for $\mathrm{CO}_{2}$ atmosphere

- Portable Life Support System (PLSS): Evaluate existing technologies for use in $\mathrm{CO}_{2}$ atmosphere, may need to develop a new PLSS schematic

- Power-Avionics-Software: increase the capabilities of the information system for additional autonomy (even bigger time delay); take advantage of advances in battery or avionics components as appropriate

\section{Long Duration Spaceflight Medical Care}

\section{Life Sciences/HRP (OCT TA 6.3)}

Description

- Strong evidence from spaceflight and analogs indicate that medical conditions of different complexity, severity, and emergency will inevitably occur during long-term Exploration missions. Long duration missions ( $>1 \mathrm{yr}$ ) increase the risk of serious medical conditions due to limited options for return to Earth, no resupply, highly limited mass, volume and some communication delays. Plans for medical care consider the most likely medical conditions, their operational and health consequences and the resources needed for treatment. Plans for the medical system seek to minimize the probability of mission failure or loss of crew.

- HRP's Integrated Medical Model (IMM) simulates medical events during space flight missions and estimates the impact of these events on crew health and mission success. A three-crew, 386 day, asteroid mission simulation with 28, 2-crew EVAs suggests an optimized medical kit having a mass of $62 \mathrm{~kg}$ and a volume of $0.15 \mathrm{~m}^{3}$. (These figures do not include all of the medical equipment needed for diagnosis).

- The medical system must monitor and treat crewmembers during the mission. The requirements for the medical system are impacted by mission duration; number of EVAs; age and gender of the crew; and crew medical expertise

- The return of biological samples is required to assess human system response to the mission in order to efficiently mitigate risks in future missions.

- Technologies will be tested on ISS and in flight analog environments

Performance characteristics

- Rapidly evolving technologies in this area will be developed to help select and prepare crew and optimize care during the mission.

- Platforms that integrate multiple diagnostic and therapeutic smart medical devices, focusing on early detection and intervention of high-consequence and remediable conditions, with consideration for dual-use technologies. Capabilities include: diagnostic imaging, oxygen concentrator, ventilator, laboratory analysis (saliva, blood, urine), bone fracture stabilization and healing, medical suction, rapid vascular access, dental care, kidney stone diagnosis and treatment, IV solution preparation and delivery, medical consumables inventory tracking, and medical data management.

\section{Long-Duration Spaceflight Behavioral Health and} Performance

\section{Life Sciences/HRP (OCT TA 6.3)}

Description

- Behavioral health and interpersonal relations among crewmembers are critical to the success of long duration exploration missions in isolated, confined and extreme environments. Technologies are required for crew selection and composition, training, support, monitoring, and intervention.

\section{Performance characteristics}

- The habitable volume must be large enough and laid out to execute the necessary tasks and to provide a psychologically acceptable space for the long period of confinement. 
- Sensory stimulation (e.g., variable lighting, virtual reality) must be augmented to offset the physically and socially monotonous environment.

- Cognitive performance deficits, stress, fatigue, anxiety, depression, behavioral health, task performance, teamwork, and psychosocial performance must be unobtrusively monitored.

- Devices must mitigate the effects of fatigue, circadian misalignment, work-overload.

- Communication tools must offset communication delays ranging from seconds to minutes.

Microgravity Biomedical Countermeasures for Long Duration Spaceflight

\section{Life Sciences/HRP (OCT TA 6.3)}

Description

- Prolonged exposure to weightlessness deconditions bone, muscle, and the cardiovascular system. Other physiological systems (e.g., sensorimotor and immune) are also altered. These changes may cause decrements in both health and performance. Countermeasures must mitigate these changes with limited resources (mass, power, volume).

- A recently discovered health risk, On-Orbit Intracranial Hypertension, would limit missions to six months or less. $20 \%$ of long duration ISS crewmembers have experienced clinical symptoms; some of these changes were temporary and others have been, to date, permanent.

\section{Performance characteristics}

- Assess sensorimotor function within 20 min with a portable hand-held device that also provides rehabilitation.

- Integrate multiple diagnostic and therapeutic smart medical devices, focusing on early detection and intervention of high-consequence and remediable conditions, with one platform

- Non-invasively measure intracranial pressure

- Worst case solution for On-Orbit Intracranial Hypertension: Artificial gravity would be required.

Microgravity Biomedical Countermeasures-Optimized Exercise Equipment

\section{Life Sciences/HRP (OCT TA 6.3)}

Description

- Exercise equipment is necessary to address muscle atrophy, cardiovascular atrophy, and bone loss associated with long-duration missions in the weightless environment of space.

- Current ISS exercise equipment is too large and heavy to be used on a long duration missions ( 1 yr duration): the latest equipment deployed on ISS occupies 3 International Standard Payload Racks.

\section{Performance characteristics}

- Provide integrated aerobic and resistive exercises with a device no larger than $45-$ by $25-$ by $25-\mathrm{cm}$, with a mass of no more than $5.4 \mathrm{~kg}$, requiring no external power, and accommodating a range of motion of at least $1 \mathrm{~m}$.

- Assess the quantity and quality of bone and muscle at multiple times over the course of a long-duration space mission

\section{Deep Space Mission Human Factors and Habitability}

Life Sciences/HRP (OCT TA 6.3, 6.1)

Description

- Human factors technologies are required in design and operations planning to ensure adequate human performance, reduce likelihood of human errors, and increase mission safety.

- Technologies are required in the habitable volumes (e.g., suit, capsule, habitat, exploration vehicle, lander) to provide an adequate food system, and to meet human environmental standards for air, water, and surface contamination.

Performance characteristics

- Onboard decision support tools assist crew with realtime detection and diagnosis of vehicle and habitat operational anomalies

- In-situ capability to assist the crew with contingency mission planning and development and execution of contingency operational procedures

- Ground-based decision support tools assist crew with mission operational anomalies with stale telemetry and operationally significant communications delays

- Reduce food packaging volume (30\%) and mass (34\%) so that supplies for one crew member for $1 \mathrm{yr}$ require $440 \mathrm{~kg}$ and $1.2 \mathrm{~m}^{3}$ consistent with food shelflife requirements, especially for long duration missions.

- An EVA suit injury countermeasures garment protects against injury caused by hard points in the suit and minimize movement of the crewmember within the volume of the suit. The garment protects the arms, legs, and torso.

- The EVA suit supports delivery of nutrition and medication to suited crew

- Microbial and chemical contamination are identified and measured in real-time with minimal resupply

\section{In-Flight Environmental Monitoring}

Life Support (OCT TA 6.4, 11)

Description

- Extended duration missions from beyond low Earth orbit will require autonomous capabilities for environmental monitoring to assess the habitation environment and recycled life support consumables and to enable the crew to anticipate, react, and mitigate any risks to continued human occupancy.

Performance characteristics

- In-flight analysis capabilities are necessaryReturning samples to Earth for ground analysis will not be feasible for future missions. Environmental habitat problems on ISS are solved by sending air and 
water samples to Earth for lab analysis, which yields data for diagnosing the problems.

- Rapid detection of hazardous environmental events must be monitored and controlled with high accuracy. Chemical (whether predicted or not) hazards are highest in urgency, followed by microbiological threats, based on rapidity of impact.

- Detect contaminants introduced via surface activities (dust, etc.) and of importance to planetary protection.

- Air Monitoring is well developed the system size should be reduced. Some specific tests for chemicals in water and for microorganisms have been flown, but analysis needs must be specified and developed.

\section{Space Radiation Protection-Galactic Cosmic Rays}

\section{Life Sciences/HRP (OCT TA 6.5)}

Description

- Current estimates of crew risk from GCR radiation exposure with long duration ( >1 yr) missions beyond LEO exceed the NASA acceptable career standards for Risk of Exposure Induced Death for fatal cancers. In many cases, the risk estimates (Cancer Risk Projection Model currently under review with National Academy of Science) greatly exceed the acceptable limit.

- Research indicates that mortality risk from radiation induced degenerative disease may further exacerbate the problem. GCR is difficult to shield against due to its high charge and energy, however shielding systems must minimize exposure levels to the maximum extent practical.

- In addition, there are large associated uncertainties in the modeling of the biological damage caused by GCR. These uncertainties limit our ability to accurately evaluate risks and the effectiveness of biological and physical mitigation strategies.

\section{Performance characteristics}

- Technological approaches include: risk quantification and uncertainty reduction through radiobiology research, selection of crew based on individual sensitivity for major risks, new biomedical countermeasures, cost/mass efficient multi-use shield systems, and mission planning away from solar minimum.

\section{Space Radiation Protection-Solar Particle Events (SPE)}

\section{Life Sciences/HRP (OCT TA 6.5) Description}

- Shielding from solar particle events (SPEs) is much easier than shielding from GCR and is required to mitigate the risk of early Acute Radiation Syndromes as well as increased risk of late radiation carcinogenesis. Protecting humans from SPEs may be a solvable problem in the near-term through technology maturation of identified shielding solutions, through design and configuration. However, mission operational planning has a major knowledge gap of forecasting the occurrence and magnitude, as well as all clear periods, of SPEs. NASA's radiation exposure standards permit a $3 \%$ risk of radiation exposure induced death. This standard limits mission durations at solar minimum to 5 to 6 months for males and approximately 3 months for females. At solar maximum, the recommended limits become 154 days for 35-year old females to 300 days for 55-year old males.

- Management of the risk of exposure to SPEs requires an overall risk model, SPE forecasting for mission planning, SPE warnings and alerts to change mission planning, shielding options for the crew under different operational scenarios, in-mission dosimetry readings, and biological countermeasures to mitigate exposures.

Performance characteristics

- Risk projection models

- Forecasting/probabilistic models of events and allclear periods

- Heliospheric environmental monitoring technology that provides accurate alerts for SPEs

- Multi-functional SPE shield systems including shelters

- Active miniaturized dosimetry

- Acute biological countermeasures

\section{TA 07 HUMAN EXPLORATION DESTINATION}

\section{SYSTEMS}

Technology advancements with In-Situ Resource Utilization to produce fuel, $\mathrm{O} 2$, and other resources, improved mobility systems including surface, off-surface and Extravehicular Activity and Extravehicular Robotics, advanced habitat systems, and advancements in sustainability and supportability technologies.

\section{Lunar ISRU: Oxygen/Water Extraction From Regolith}

\section{ISRU (OCT TA 7.1)}

\section{Description}

- In-situ resource utilization (ISRU) involves the extraction and processing of local resources, both natural and discarded, into useful products and services. In particular the extraction of oxygen, water, and other volatiles that can be used for life support, propellants, fuel cell power systems, and radiation protection can significantly reduce the mass, cost, and risk of short term and sustained human exploration of the Moon. Lander reusability and in-space propellant depots for Cis-lunar transportation are enabled. The two lunar ISRU products and processes that have the biggest impact on human mission architectures are:

- Oxygen extraction from lunar regolith: This involves excavation of loosely consolidated surface regolith, regolith transfer and handling (size sorting and mineral beneficiation), and chemical/thermal processing to remove oxygen from mineral oxides. The Moon is $\sim 42 \%$ oxygen by mass. Operations occur in nominal lunar day/night cycle conditions. 
- Water and volatile extraction from lunar polar regolith: This involves first locating and characterizing lunar polar ice/volatile deposits, then excavation (down to $1 \mathrm{~m}$ possible), regolith transfer and handling (possibly crushing), heating to evolve water and volatiles, and volatile capture and separation. Operations may occur at extremely low temperatures (40 to $100 \mathrm{~K}$ ).

\section{Performance characteristics}

- Pilot plant to produce oxygen from lunar regolith; 250 to $500 \mathrm{~kg}$ per year; Extraction efficiency $>1 \%$ oxygen by weight; Mass Payback (break-even point) is $<1 \mathrm{yr}$ compared to bringing oxygen from Earth, considering the mass of a complete ISRU system (excavator, plant, power system, and storage system).

- Full Scale plant to produce oxygen from lunar regolith: 1000 to $10,000 \mathrm{~kg}$ per year (depending on crew size and propellant need); Mass Payback (breakeven point) is $<1 \mathrm{yr}$ compared to bringing oxygen from Earth, considering the mass of a complete ISRU system (excavator, plant, power system, and storage system).

- Water Extraction Plant from polar regolith: TBD. Water usage as well as currently unknown polar water/ice concentration significantly influence metrics.

Mars ISRU: Oxygen from Atmosphere and Water Extraction from Soil

ISRU (OCT TA 7.1)

Description

- In-situ resource utilization (ISRU) involves the extraction and processing of local resources, both natural and discarded, into useful products and services. In particular the production of oxygen, water, and methane that can be used for life support, propellants, fuel cell power systems, and radiation protection can significantly reduce the mass, cost, and risk of short term and sustained human exploration of Mars. The two Mars ISRU products and processes that have the biggest impact on robotic sample return and human Mars mission architectures are:

- Oxygen production from Mars atmosphere $\mathrm{CO}_{2}$ : This involves the collection and separation of carbon dioxide $\left(\mathrm{CO}_{2}\right)$ from the 6 to 10 torr Mars atmosphere and processing the $\mathrm{CO}_{2}$ to extract oxygen. Oxygen can make up $>75 \%$ of propellant mass.

- Oxygen and fuel production from Mars soil water and atmosphere $\mathrm{CO}_{2}$ : This involves excavation of Mars soil and processing/heating to release water. Water is electrolyzed to make oxygen and hydrogen (for processing). This also involves collection and separation of carbon dioxide $\left(\mathrm{CO}_{2}\right)$ from the 6 to 10 torr Mars atmosphere and processing with hydrogen to make methane (or other hydrocarbon) and water.

\section{Performance characteristics}

- Atmospheric $\mathrm{CO}_{2}$ processing; $3.5 \mathrm{~kg} \mathrm{O} / \mathrm{hr}$ and $1 \mathrm{~kg}$ $\mathrm{CH}_{4} / \mathrm{hr}, 24 \mathrm{hr} /$ day, $300 \mathrm{~d}$. $<7 \mathrm{KWe} / \mathrm{kg} \mathrm{O}_{2}$ produced.
- Water extraction from soil: $2 \mathrm{~kg} \mathrm{H} 2 \mathrm{O} / \mathrm{hr}, 24 \mathrm{hr} /$ day, 300 d. $\sim 40 \mathrm{~kg}$ soil/hr excavation and processing. <15 $\mathrm{KWe} / \mathrm{kg}$ water extracted.

Anchoring Techniques and EVA Tools for Micro-G Surface Operations

EVA (OCT TA 7.3)

Description

- Anchoring/mobility for a NEO mission, Exotic Geology Sample Acquisition, Real time Geology Sample Analysis

Performance characteristics

- Anchoring techniques for vehicles and EVA systems are needed for asteroid missions

- ISS uses well defined interfaces such as hand rails as opposed to unknown rocky surfaces

- The ability to collect geological samples without damaging the sample (minimal heat or stress) or from a location with difficult access (bottom of a crater or top of a cliff) is needed

- Increased ability to analyze the chemical or physical properties of samples collected maximizes the useful data collected and minimizes the need to bring samples back to Earth

- All tool development must consider environmental factors and EVA compatibility (safety, mobility limitations)

\section{Suit Port}

EVA (OCT TA 7.3)

Description

- A suit port provides a method of rapidly starting and ending EVAs and provides an increased level of environmental containment of potentially hazardous substances that could be encountered during the EVA.

Performance characteristics:

- Reduce airlock operations time from $4 \mathrm{hr}$ pre- and post-EVA to $30 \mathrm{~min}$

- Reduce exposure of habitable volume to dust, particulates, heat transport fluids, propellants, gases such as atmospheric $\mathrm{CO}_{2}$, etc.

- Reduce consumable losses from habitable volume by $660 \mathrm{~kg}$ over two weeks (assumes multiple EVAs/day)

\section{Surface Mobility}

\section{Robotics and Mobility (OCT TA 7.3)}

Description

- Surface mobility systems allow for the movement of cargo, instruments and crew on the surface of an object or planetary body. Examples include roving, climbing, crawling, hopping or burrowing into the surface. Systems for moving cargo include prepositioning cargo for future human use, or repositioning payloads for re-use. Instruments can be pointed by mobility systems, or pushed into contact for data collection, approaching simple manipulation by using the mobility system's transport mechanisms. Crew mobility aids expand crew range, speed and payload capacity while also providing power, 
habitation and environmental shelter. NASA's experience with crew mobility on the lunar surface was limited to unpressurized rovers for short stays. NASA now faces new challenges of working on the exteriors of satellites, on asteroid surfaces, on planetary surfaces for long durations, or providing access to lunar craters. Complexities of dust management and human interaction with NEA during extended should also be addressed.

Performance characteristics

- Microgravity climbing for satellite or asteroid missions

- Precursor roving in soft/steep soils for lunar crater access

- Ballistic crater explorer, fires projectile into crater for data

- Concurrent design of crew rover and SEV for re use

- Mobile landers for repositioning spacecraft on small bodies

\section{Mission Control Automation Beyond LEO}

\section{Avionics and Software (OCT TA 7.5, 4.7) \\ Description}

- Support Missions beyond LEO in problem solving activities during remote or long-duration exploration missions, where space crew reliance on mission control is critical and dependent upon minimum reaction time. Advanced decision-support systems are needed in Mission Control to reduce operations costs and to maximize mission safety with Earth-based operators.

\section{Performance characteristics}

- Enable Earth-based nominal operation of vehicle or habitat at destinations with $>6$ s round-trip time delay to Earth

- Enable hand-offs in Mission Ops between ground and crew for operations in transit and at destinations with $>6$ s round-trip time delay

- Enable Tools to help Flight Controllers resolve off nominal situation after detection and initial response

- Enable highly efficient, small staff Earth-based Mission Control for Beyond LEO Crewed Missions

\section{Dust Mitigation}

\section{Space Environment (OCT TA 7.5)}

Description

- Technologies are required to address adverse regolith effects in order to reduce life cycle cost and risk, and increase the probability of mission success. Based on Apollo lunar surface experience, there is a risk of regolith induced system degradation. The NEO environment may include suspended "clouds" of particulates, and is in any case an unknown. Particulate mitigation will be accomplished by:

- Identification of NEO soil contamination issues for mechanisms and thermal systems.

$\circ$ Investigate specific risk mitigation technologies (e.g., seals) applicable to NEO missions. Develop technologies to limit regolith contamination, or mitigate its effects.

- In a relevant environment, integrate and test mechanical component-level technologies to TRL 6.

- NEO simulants are required to develop tools for anchoring, sample acquisition, etc, and Mars simulants are needed to develop ISRU technology.

- Regolith dust self-cleaning radiators needed for surface operations.

- Dust tolerant components or self cleaning capability is needed for Lunar Surface Space Suits (Block 2).

- Active dust removal technology (SPARCLED) can also be used to acquire small-sized samples from NEOs or dust-sized samples from reduced-gravity bodies.

Performance characteristics

- Mitigation technologies must:

- Maintain the solar absorptivity of a dust contaminated radiator surface within $+20 \%$ of the pristine surface value, and

- Provide negligible dynamic seal wear to 2 million cycles (approx. 6 month life) or 20 million cycles for a $5 \mathrm{yr}$ life.

TA 09 ENTRY, DESCENT, AND LANDING SYSTEMS Human-class capabilities for Mars entry, descent, and landing; technologies advancing low mass high velocity Thermal Protection Systems (TPS), atmospheric drag devices, deep-throttling engines, landing gear, advanced sensing, aero-breaking, aero-capture, etc. Soft precision landing capability is also needed, e.g., for Moon and NEA's.

Entry, Descent and Landing (EDL) Technologies- Mars Exploration Class Missions

EDL (OCT TA 9.1, 9.2, 9.3, 9.4)

Description

- Entry, descent and landing systems for Mars exploration-class missions require large surface payloads. This technology enables reliable and safe delivery of multiple $40 \mathrm{mt}$ payloads to the surface of Mars in order to support human exploration. The benefits of focused EDL technology activities include: increased mass delivery to a planet surface (or deployment altitude), increased planet surface access (both higher elevation and latitudes), increased delivery precision to the planet's surface, increased robustness of landing system to surface hazards, and enhanced safety and probability of mission success for EDL phases of atmospheric flight..

\section{Performance characteristics}

- Aeroassist, Aerocapture, and Entry-AAES are defined as the intra-atmospheric technologies that decelerate a spacecraft from hyperbolic arrival through the hypersonic phase of entry. Options include deployable, inflatable, and mid-L/D vehicles, which need to be actively guided to limit loads and achieve accurate landings. 
- Descent-These technology advancements primarily focus on providing greater deceleration in the supersonic and subsonic regimes in a manner that does not reduce landing accuracy or result in transient unsteadiness or loss of performance in the transonic regime. For human-class missions, inflatable and retropropulsion technologies are options.

- Landing - The key areas of technology development are the systems to sense the surface and avoid hazards, descent propulsion motors and plume-surface interaction mitigation, touchdown systems, high-G survivable systems, and small-body guidance. Landed payloads include: Large Robotic Landers (100 to 1500 $\mathrm{kg}$ ) and Human Class (1500 to $45000 \mathrm{~kg}$ )

- Vehicle Systems-EDL systems are by their nature an integrated framework of technologies that necessitate system level validation for robust maturation.

- Modeling and simulation along with atmospheric and surface characterization activities are essential for advancing these technologies.

Entry, Descent and Landing (EDL) Technologies- Earth Return

EDL (OCT TA 9.1, 9.2, 9.3)

Description

- Earth Return entry, descent and landing systems for Human exploration architecture missions include high-velocity (8 to $14 \mathrm{~km} / \mathrm{s}$ ) Earth entries from beyond LEO - from HEO, NEAs, libration points, the Moon, and Mars. This technology enables reliable and safe return of crew and/or logistics, and may have reusability requirements. The benefits of focused Earth return technology activities include: human safety during return from missions beyond LEO, lower-mass return capsules, increased landing system robustness, enhanced safety and probability of mission success, architecture flexibility and element reusability, and for robotic missions, sample return reliability and planetary protection. Technology developments must begin immediately in order to enable early exploration architectures. Extensive ground testing and flight tests in Earth's atmosphere will be necessary to meet reliability requirements.

\section{Performance characteristics}

- Aeroassist, Aerocapture, and Entry- AAES are defined as the intra-atmospheric technologies that decelerate a spacecraft from hyperbolic arrival through the hypersonic phase of entry. Ablative materials are an enabling technology needed for high velocity entries $(>8 \mathrm{~km} / \mathrm{s}$, up to $16 \mathrm{~km} / \mathrm{s}$ for robotic comet sample return missions), possible aerocaptures for reusability or skip entries for downrange capability, and possible dual heat pulse entries. Keys are low-cost, high-reliability manufacturing and subsystem/system performance modeling and validation.

- Descent-At Earth, these are usually parachutes; systems for this flight regime could have increased requirements due to higher entry velocities. For sample return capsules, inherently stable vehicles without parachutes are preferred to meet the reliability requirements for minimal mass.

- Landing - The key area of technology development is the impact attenuation system; some large-system progress has been made through Orion (sample return capsules will likely have different requirements)

- Vehicle Systems-EDL systems are by their nature an integrated framework of technologies that necessitate system level validation for robust maturation.

- Modeling and simulation are essential for quantifying the reliability of these systems.

TA 11 MODELING, SIMULATION, INFORMATION TECHNOLOGY AND PROCESSING

Advancements in technologies associated with flight and ground computing, integrated $\mathrm{s} / \mathrm{w}$ and $\mathrm{h} / \mathrm{w}$ modeling systems, simulation and information processing.

\section{Advanced Software Development/Tools}

\section{Avionics and Software (OCT TA 11.2) \\ Description}

- Reliable software engineering tools and technologies to ensure system reliability and reduce software costs (and hence system and mission costs).

\section{Performance Characteristics}

- Increase software design productivity and reduce lifecycle software DDT\&E and maintenance costs, greatly lowering \$cost/SLOC (source line of code)

$\circ$ Qualification of model-based software development methods

- Dynamic certification/recertification of software developed through model-based and other highly automated methods

- Software system infrastructure to leverage multicore avionics

- Reusable software platforms suitable for humanrated spaceflight

- Ensure on-board software reliability for long-duration human missions with light-time delay

- Enable verification of advanced software-based functions for: crew autonomy, autonomous systems, vehicle systems health management, and situational awareness capabilities

TA 12 MATERIALS, STRUCTURES, MECHANICAL SYSTEMS AND MANUFACTURING

Technology advancements for lightweight structures providing radiation protection, multifunctional structural design and innovative manufacturing. In addition, new technologies associated with reducing design, manufacturing, certification and life-cycle costs.

\section{Structures and Materials for Inflatable Modules}

Structures/Materials (OCT TA 12.1, 12.2, 7.4.2)

\section{Description}

- The primary advantage of inflatable/expandable structures is the readily collapsible walls that reduce stowage volume for the launch package, but provide extra volume for living space when expanded. The 
resulting mass-to-volume ratio for expandable structures can be lower than that for conventional hard shell structures.

- The objective is to develop expandable structures technology for application as pressurized elements such as crew habitats, logistics add-ons, and airlocks. The goal is to develop expandable technology for increased deployed-habitable volume for minimal packing volume, with improved confidence in structural and thermal performance in the space environment.

Performance characteristics

- Long-term creep performance characterization of the structural shell of the inflatable module

- Inflatable Structure Restraint Layer damage tolerance (predictive modeling validated with testing).

- Multi-layer insulation performance degradation prediction after folding/deployment (predictive modeling validated with testing).

- Bladder material selection.

- Bladder-to-metal interface seal.

- Predictive modeling of deployment dynamics.

\section{Lightweight and Efficient Structures and Materials}

Structures/Materials (OCT TA 12.1, 12.2)

Description

- Efficient Structures and Materials that demonstrate significant weight and cost savings for aerospace applications to provide a total systems based efficiency. This includes multifunctional, lightweight and robust (i.e., inspectable, repairable, damage tolerant, etc.) structures and materials specifically tailored for mission applications.

- Emerging Innovations in Manufacturing Technology that offer significant improvement over SOA, critical to achieving the destination, performance, and affordability objectives for exploration

- Design and Certification Methods to ensure timely introduction of advanced, multifunctional structures and materials into future reliable space systems

- Damage models for reliability (certification and sustainment)

- Optimized analysis and test for verification and validation

- Streamlined Design-Analysis-Certification processes

- Rapid material properties development

Performance Characteristics

- Lightweight structures and materials optimization to realize structural system dry mass savings (minimum of 20 to $25 \%$ ) and operational cost savings.

- Multifunctional structures that offer improvements in radiation protection, MMOD shielding, thermal management, structural health management, and system damping benefits over conventional structures. Includes composite and metallic materials.

TA 13 GROUND AND LAUNCH SYSTEMS PROCESSING
Technologies to optimize the life-cycle operational costs, increase reliability and mission availability, improve mission safety, reduce mission risk, and reduce environmental impacts (i.e.., green technologies).

HAT Technology Needs in this technology area do not map to JSC Core Technology Competencies. See [9] for details of HAT technologies in this discipline area.

\section{TA 14 THERMAL MANAGEMENT SYSTEMS}

Technology advancement for cryogenic systems performance and efficiency, effective thermal control systems for heat acquisition/transport/rejection, and increased robustness and reduced maintenance for thermal protection systems

\section{Thermal Control}

Thermal Systems (OCT TA 14.2)

Description

- All future vehicles (both crewed and uncrewed) will require thermal control systems (TCS)

- Improve thermal control system performance and reliability to reduce mass transportation requirements and enable performance over a wide range of mission requirements.

- Thermal control in day/night with dust mitigation on radiators is critical for continuous ops and survival.

- Technologies that will be required include:

- TCS fluids and variable heat rejection radiators enabling single-loop TCS architecture

- Low mass/volume heat exchangers and coldplates

- Advanced Supplemental Heat Rejection Devices including evaporative heat sinks and fusible heat sinks

- Solid state devices (thermal electrics) and thermal sensors/health monitoring

- Operations in Lunar Perennially Shadowed Regions at Cryogenic Temperatures (40 K)

Performance Characteristics

- Capable of maintaining system setpoint for large turndown ratio requirements (12 to $1 \mathrm{~kW}$ )

- Exacerbated by low load in cold environment $(\sim 0$ $\mathrm{K})$ and high load in hot environment $(\sim 220 \mathrm{~K})$

- Capable of efficient operation in rapidly changing thermal environments and/or transient heat rejection requirements

- Reduces component and system mass

Robust Ablative Heat Shield (beyond Lunar return conditions) - Thermal Protection System

\section{Structures and Materials (OCT TA 14.3)}

Description

- A robust, scalable heat shield TPS architecture is required that can be used for multiple missions. Ablative TPS solution for primary MPCV heat shield protection for beyond Lunar return conditions. Improve human safety by detecting critical issues with MPCV TPS or structure prior to entry. 
Performance characteristics

- Ablative TPS Solution for primary CTV heat shield capable of withstanding $\sim 2500 \mathrm{~W} / \mathrm{cm}^{2}$ under 0.8 atmosphere pressure

- Peak heat rate dominated $(\sim 90 \%)$ by shock layer radiation

- Technology needs to enter DDT\&E cycle including TPS development, aerothermal and shock layer radiation modeling validation, reliability/margin quantification methodology, integrated system health monitoring, and hyperthermal ground test capability to approximate convective-radiative environment.

Robust Ablative Heat Shield (Lunar return conditions)_-Thermal Protection System

Structures and Materials (OCT TA 14.3)

Description

- A robust, scalable heat shield TPS architecture is required that can be used for multiple missions. Ablative TPS solution for primary MPCV heat shield protection. Improve human safety by detecting critical issues with MPCV TPS or structure prior to entry.

\section{Performance Characteristic}

- Capable of withstanding $\sim 1000 \mathrm{~W} / \mathrm{cm}^{2}$ (about $33 \%$ radiation) and $\sim 1$ atmosphere pressure

\section{APPENDIX B: ACRONYMS}

$\begin{array}{ll}\text { AAES } & \text { Aeroassist, Aerocapture, and Entry Systems } \\ \text { AR } & \text { Atmospheric Revitalization } \\ \text { AU } & \text { Astronomical Unit } \\ \text { BAC } & \text { Broad Area Cooling } \\ \text { BPP } & \text { Bubble Point Pressure } \\ \text { CDR } & \text { Critical Design Review } \\ \text { CFM } & \text { Cryogenic Fluid Management } \\ \text { CH4 } & \text { Methane } \\ \text { COTS } & \text { Commercial Off the Shelf } \\ \text { CPS } & \text { Cryogenic Propulsion Stage } \\ \text { CTV } & \text { Crew Transport Vehicle (aka MPCV) } \\ \text { CXP } & \text { Constellation Program } \\ \text { DDT\&E } & \text { Design, Development, Test and Evaluation } \\ \text { DRA } & \text { Design Reference Architecture } \\ \text { DRM } & \text { Design Reference Mission } \\ \text { DSH } & \text { Deep Space Habitat } \\ \text { DSN } & \text { Deep Space Network } \\ \text { ECLS } & \text { Environmental Control and Life Support } \\ \text { EDL } & \text { Entry, Descent, Landing } \\ \text { E-M } & \text { Earth-Moon } \\ \text { EVA } & \text { Extravehicular Activity } \\ \text { FDIR } & \text { Fault Detection, Fault Isolation, and Recovery } \\ \text { GCR } & \text { Galactic Cosmic Rays } \\ \text { GEO } & \text { Geosynchronous Earth Orbit } \\ \text { HAT } & \text { Human Space Flight Architecture Team } \\ \text { HEFT } & \text { Human Exploration Framework Team } \\ \text { HEO } & \text { High Earth Orbit } \\ \text { HEOMD } & \text { NASA's Human Exploration and Operations } \\ & \text { Mission Directorate } \\ \text { HRP } & \text { NASA's Human Research Program } \\ & \end{array}$

HSF Human Space Flight

ICA Innovative Charge Account

IMM Integrated Medical Model

IR\&D Internal Research and Development

ISCEG International Space Exploration Coordination Group

ISRU In-Situ Resource Utilization

ISS International Space Station

IV Intravenous

IVA Intravehicular Activity

L1 Lagrange Point 1

L2 Lagrange Point 2

LAD Liquid Acquisition Device

Lbf Pound-force

LCH4 Liquid Methane

LEE Latching End Effectors

LEO Low Earth Orbit

LH2 Liquid Hydrogen

LLO Low Lunar Orbit

LO2 Liquid Oxygen

LOx Liquid Oxygen

MCC Mission Control Center

MLI Multilayer Insulation

MMOD Micrometeorites and Orbital Debris

MPCV Multipurpose Crew Vehicle

NASA National Aeronautics and Space Administration

NEA Near Earth Asteroid

NEO Near Earth Object

NRC National Research Council

NTP Nuclear Thermal Propulsion

OCT NASA's Office of the Chief Technologist

ORSC Oxygen-Rich Staged Combustion

PDR Preliminary Design Review

PLSS Portable Life Support System

PMAD Power Management and Distribution

PSR Perennially Shadowed Regions

RBO Reduced Boil Off

RCS Reaction Control System

REM Robotics and EVA Module

$R F \quad$ Radio Frequency

RFC Regenerative Fuel Cell

RP Rocket Propellant

SARJ Solar Alpha Rotary Joint

SEP Solar Electric Propulsion stage

STS Space Transportation System

SLOC Source Line of Code

SLS Space Launch System

SOA State of the Art

SRR Strategic Readiness Review

SPE Solar Particle Events

SSRMS Space Station Remote Manipulator System

TA Technical Area

TBD To Be Determined

TRL Technology Readiness Level

TPS Thermal Protection System

WR Water Recovery

ZBO Zero Boil Off 
